\title{
DOMINATION GAME CRITICAL GRAPHS
}

\author{
Csilla Bujtás \\ Department of Computer Science and Systems Technology \\ University of Pannonia, Veszprém, Hungary \\ e-mail: bujtas@dcs.uni-pannon.hu \\ SANDI KLAVŽAR \\ Faculty of Mathematics and Physics, University of Ljubljana, Slovenia \\ Faculty of Natural Sciences and Mathematics, University of Maribor, Slovenia \\ Institute of Mathematics, Physics and Mechanics, Ljubljana, Slovenia \\ e-mail: sandi.klavzar@fmf.uni-lj.si
}

AND

GAŠPER KOŠMRLJ

Faculty of Mathematics and Physics, University of Ljubljana, Slovenia

e-mail: gasper.kosmrlj@student.fmf.uni-lj.si

\begin{abstract}
The domination game is played on a graph $G$ by two players who alternately take turns by choosing a vertex such that in each turn at least one previously undominated vertex is dominated. The game is over when each vertex becomes dominated. One of the players, namely Dominator, wants to finish the game as soon as possible, while the other one wants to delay the end. The number of turns when Dominator starts the game on $G$ and both players play optimally is the graph invariant $\gamma_{g}(G)$, named the game domination number. Here we study the $\gamma_{g}$-critical graphs which are critical with respect to vertex predomination. Besides proving some general properties, we characterize $\gamma_{g}$-critical graphs with $\gamma_{g}=2$ and with $\gamma_{g}=3$, moreover for each $n$ we identify the (infinite) class of all $\gamma_{g}$-critical ones among the $n$th powers $C_{N}^{n}$ of cycles. Along the way we determine $\gamma_{g}\left(C_{N}^{n}\right)$ for all $n$ and $N$. Results of a computer search for $\gamma_{g}$-critical trees are presented and several problems and research directions are also listed.
\end{abstract}

Keywords: domination number, domination game, domination game critical graphs, powers of cycles, trees.

2010 Mathematics Subject Classification: 05C69, 05C57. 


\section{REFERENCES}

[1] J.A. Bondy and U.S.R. Murty, Graph Theory (Springer, New York, 2008). doi:10.1007/978-1-84628-970-5

[2] B. Brešar, P. Dorbec, S. Klavžar and G. Košmrlj, Domination game: Effect of edgeand vertex-removal, Discrete Math. 330 (2014) 1-10. doi:10.1016/j.disc.2014.04.015

[3] B. Brešar, S. Klavžar, G. Košmrlj and D.F. Rall, Domination game: extremal families of graphs for the 3/5-conjectures, Discrete Appl. Math. 16 (2013) 1308-1316. doi:10.1016/j.dam.2013.01.025

[4] B. Brešar, S. Klavžar and D.F. Rall, Domination game and an imagination strategy, SIAM J. Discrete Math. 24 (2010) 979-991. doi: $10.1137 / 100786800$

[5] R.C. Brigham, P.Z. Chinn and R.D. Dutton, Vertex domination-critical graphs, Networks 1 (1988) 173-179. doi:10.1002/net.3230180304

[6] Cs. Bujtás, Domination game on trees without leaves at distance four, in: Proceedings of the 8th Japanese-Hungarian Symposium on Discrete Mathematics and Its Applications, A. Frank, A. Recski, G. Wiener, Eds., June 4-7 (2013) Veszprém, Hungary, 73-78.

[7] Cs. Bujtás, Domination game on forests, Discrete Math. 338 (2015) 2220-2228. doi:10.1016/j.disc.2015.05.022

[8] Cs. Bujtás, On the game domination number of graphs with given minimum degree, Electron. J. Combin. 22 (2015) \#P3.29.

[9] T. Burton and D.P. Summer, Domination dot-critical graphs, Discrete Math. 306 (2006) 11-18. doi:10.1016/j.disc.2005.06.029

[10] P. Dorbec, G. Košmrlj and G. Renault, The domination game played on unions of graphs, Discrete Math. 338 (2015) 71-79. doi:10.1016/j.disc.2014.08.024

[11] M. Furuya, Upper bounds on the diameter of domination dot-critical graphs with given connectivity, Discrete Appl. Math. 161 (2013) 2420-2426. doi:10.1016/j.dam.2013.05.011

[12] M. Furuya, The connectivity of domination dot-critical graphs with no critical vertices, Discuss. Math. Graph Theory 34 (2014) 683-690. doi:10.7151/dmgt.1752

[13] M.A. Henning, S. Klavžar and D.F. Rall, Total version of the domination game, Graphs Combin. 31 (2015) 1453-1462. doi:10.1007/s00373-014-1470-9

[14] M.A. Henning, S. Klavžar and D.F. Rall, The 4/5 upper bound on the game total domination number, Combinatorica, to appear. 
[15] M.A. Henning, O.R. Oellermann and H.C. Swart, Distance domination critical graphs, J. Combin. Math. Combin. Comput. 44 (2003) 33-45.

[16] S.R. Jayaram, Minimal dominating sets of cardinality two in a graph, Indian J. Pure Appl. Math. 28 (1997) 43-46.

[17] W.B. Kinnersley, D.B. West and R. Zamani, Game domination for grid-like graphs, manuscript, 2012.

[18] W.B. Kinnersley, D.B. West and R. Zamani, Extremal problems for game domination number, SIAM J. Discrete Math. 27 (2013) 2090-2107. doi: $10.1137 / 120884742$

[19] G. Košmrlj, Realizations of the game domination number, J. Comb. Optim. 28 (2014) 447-461. doi:10.1007/s10878-012-9572-x

[20] X. Li and B. Wei, Lower bounds on the number of edges in edge-chromatic-critical graphs with fixed maximum degrees, Discrete Math. 334 (2014) 1-12. doi:10.1016/j.disc.2014.06.017

[21] L. Lovász and M.D. Plummer, Matching Theory (AMS Chelsea Publishing, Providence, RI, 2009).

[22] W. Pegden, Critical graphs without triangles: an optimum density construction, Combinatorica 33 (2013) 495-513. doi:10.1007/s00493-013-2440-1

[23] F. Tian and FJ.-M. Xu, Distance domination-critical graphs, Appl. Math. Lett. 21 (2008) 416-420. doi:10.1016/j.aml.2007.05.013

[24] D.B. West, Introduction to Graph Theory (Prentice Hall, Inc., Upper Saddle River, NJ, 1996).

Received 12 December 2014

Revised 19 March 2015

Accepted 19 March 2015 